\title{
Piezo-Resistive Silicon Thin Film Sensor Array for Biomedical Applications
}

\author{
P. Alpuim $^{1 *}$, V. Correia ${ }^{1,2}$, E.S. Marins ${ }^{1}$, J.G. Rocha ${ }^{2}$, I. G. Trindade ${ }^{3}$, S. Lanceros-Mendez ${ }^{1}$ \\ ${ }^{1}$ Center of Physics, Universidade do Minho, Campus de Azurém, 4800-058 Guimarães, \\ Portugal \\ ${ }^{2}$ Algoritmi Research Center, Universidade do Minho, Campus de Azurém, 4800-058 \\ Guimarães, Portugal \\ 3 Department of Textile Science and Technology, Universidade da Beira Interior, Rua \\ Marquês D’Ávila e Bolama, 6200 Covilhã, Portugal
}

*Corresponding author. E-mail: palpuim@fisica.uminho.pt

\begin{abstract}
N-type hydrogenated nanocrystalline silicon thin film piezoresistors, with gauge factor -28 , were deposited on rugged and flexible polyimide foils by Hot-wire chemical vapor deposition using a tantalum filament heated to $1750^{\circ} \mathrm{C}$. The piezoresistive response under cyclic quasistatic and dynamical (up to $100 \mathrm{~Hz}$ ) load conditions is reported. Test structures, consisting of microresistors having lateral dimensions in the range from 50 to $100 \mu \mathrm{m}$ and thickness of 120 $\mathrm{nm}$ were defined in an array by reactive ion etching. Metallic pads, forming ohmic contacts to the sensing elements, were defined by a lift-off process. A readout circuit for the array consisting in a mutiplexer on each row and column of the matrix is proposed. The digital data will be processed, interpreted and stored internally by an ultra low-power micro controller,
\end{abstract}


also responsible for the communication of two-way wireless data, e.g. from inside to outside the human body.

\section{Keywords}

Hot-wire CVD, nanocrystalline silicon, thin films, piezoresistance, flexible electronics, sensors

\section{Introduction}

Since the piezoresistive property of silicon was first reported by C. Smith [1] it has been used in different kinds of sensors that make use of the change of conductivity under strain to probe and measure deformation of surfaces and solids. The piezoresistive devices can be simple nand p-type resistors, field-effect N- and P-MOS transistors or p-i-n and Schottky diodes [2-4]. Usually, these devices are fabricated on Si wafers using standard lithographic techniques [3]. Doped hydrogenated nanocrystalline silicon thin films, nc-Si:H, exhibit piezoresistive behavior that is some type of average over all its randomly oriented crystallites of the piezoresistrive response of a single $\mathrm{Si}$ crystal. The effect is expected to be weaker than in c-Si since only those crystallites with the proper orientation relative to the principal axis of the applied stress will contribute strongly to the overall effect. In the line of this reasoning, n-type nc-Si:H films have negative gauge factor $G F \sim-30(G F$ is the proportionality constant relating relative resistance change and applied strain) $[5,6]$ reminiscent of the larger in absolute value n-type c-Si $\pi$-coefficient: $\pi_{11}=-102 \times 10^{-11} \mathrm{~Pa}^{-1}$ ( $\pi$-coefficients are defined as the relative change in resistivity per stress) [1]. 
Hot-wire chemical vapor deposition has proved to be a very adequate technique to deposit hydrogenated nanocrystalline silicon thin films, nc-Si:H, due to its high efficiency in breaking $\mathrm{H}_{2}$ molecules into atomic hydrogen that diffuses from the heated tungsten or tantalum filament to the growing film surface where it very effectively promotes crystalline growth [7]. Although nc-Si:H has a much lower carrier mobility than c-Si due to carrier scattering at grain boundaries [8], it can, however, be deposited at low temperatures on virtually any type of substrate that stands the deposition temperature. In the present work we use polyimide plastic foil as substrate and deposit $\mathrm{n}^{+}$-nc-Si:H by HWCVD at a temperature of $150{ }^{\circ} \mathrm{C}$ thus obtaining a flexible composite of an isolating polymer covered by a piezoresistive semiconductor. Previous work has shown that, as far as piezoresistance is concerned, nc-Si:H films are isotropic in planes perpendicular to the growth direction [9]. The combined characteristics of flexibility, piezoresistance and isotropy allow the design of novel types of strain or shape sensing devices, for example for biomedical applications. It is shown that large-area flexible thin-film Si piezoresistors deposited by HWCVD on plastic substrates can respond and survive to quasi-static as well as to dynamical loading conditions up to at least hundreds of Hertz. The fabrication of micro-devices using standard lithographic techniques with minor adaptations due to the chemistry of the plastic substrates is demonstrated. Finally, the control electronics for data acquisition using an array of resistors is proposed. Data processing, storage and communication are also addressed.

\section{Experimental}

\subsection{HWCVD deposition}


Depositions were performed in a load-locked research chamber under high-vacuum conditions (base pressure better than $10^{-6}$ Torr). After loading, before starting deposition and while heating the substrates to the deposition temperature of $150^{\circ} \mathrm{C}$, the time necessary for the pressure to recover to the pre-load values was allowed (this could take tens of minutes due to the long degassing time of the polyimide foils). A single S-shaped tantalum filament, $0.5 \mathrm{~mm}$ thick and $14 \mathrm{~cm}$ long, was heated up to $1750^{\circ} \mathrm{C}$. Filament-to-substrate distance was $7 \mathrm{~cm}$. The filament was first heated up to the working temperature, in a hydrogen atmosphere, and then the source gases, $\mathrm{SiH}_{4}$ and $\mathrm{PH}_{3}$ (for gas phase P-doping), were added while the hydrogen flow was adjusted to a value corresponding to $95 \%$ of the total gas flow. Working gas pressure was 40 mTorr for all depositions. During the filament heating time, a shutter was closed in order to protect the substrate from spurious species emitted from the filament. Deposition rate was $\sim 1.5$

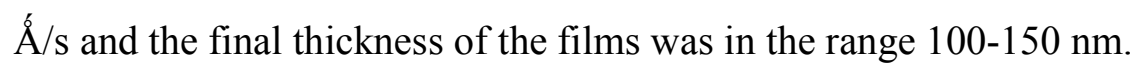

\subsection{Sensor array fabrication}

nc-Si:H films deposited on square shaped (side length $=35 \mathrm{~mm}$ ) polyimide (PI) flexible substrates with thickness $d_{s}=125 \mu \mathrm{m}$ were patterned by photolithography into strain sensing microresistors in an array with seven lines and four columns. The PI substrates were glued with photoresist (PR) to a 2" Si carrier wafer for processing. Each sensing element consists of rectangular islands with lateral dimensions (length $\times$ width) in the range from $210 \mu \mathrm{m} \times 70 \mu \mathrm{m}$ to $450 \mu \mathrm{m} \times 150 \mu \mathrm{m}$, connected to metallic leads at each end of the island and extending to large pads $(3 \mathrm{~mm} \times 2 \mathrm{~mm})$. Two masks were used to define the sensors: first, a bright field

mask defined the semiconductor islands pattern; second, a dark field mask defined the 
electrically conductive leads and pads in a lift-off process, using a positive PR (AZ Electronic Materials $\mathrm{GmbH})$.

A reactive ion etching (RIE) step was used to etch the nc-Si:H islands (etch rate $\sim 28 \mathrm{~nm} / \mathrm{min}$ ) on the PI substrate. Under the processing conditions used (pressure $=55 \mathrm{mTorr}$, Power $=150$ $\mathrm{W}, \mathrm{CHF}_{3}$ flow $=50 \mathrm{sccm}, \mathrm{SF}_{6}$ flow $=5 \mathrm{sccm}$ and $\mathrm{O}_{2}$ flow $\left.=5 \mathrm{sccm}\right)$, the Si etch rate was approximately $28 \mathrm{~nm} / \mathrm{min}$.

The metallic leads and pads, consisting of a tri-layered film of Ta $10 \mathrm{~nm} / \mathrm{Al} 40 \mathrm{~nm} / \mathrm{Ta} 5 \mathrm{~nm}$, were deposited by ion beam deposition followed by lift-off in acetone soak.

Large-area piezoresistors with a similar structure to that of the individual microresistors were fabricated using shadow masks in a four lines by two columns matrix. The piezoresistor dimensions are set by the $1 \mathrm{~mm}$ gap between the contacts, the width of the island $(3 \mathrm{~mm})$ and the thickness of the films.

\section{Results and discussion}

Figure 1 shows the piezoresistive response, under loading by 4-point bending cycles (Shimadzu-AG-IS $500 \mathrm{~N}$ ), of one nc-Si:H microresistor with thickness $120 \mathrm{~nm}$, belonging to a larger array fabricated on a $125 \mu \mathrm{m}$ thick polyimide substrate. Fig.1-a) shows the microresistor width $\times$ length dimensions of $100 \times 100 \mu \mathrm{m}^{2}$. The bright parts of the optical micrograph are the metallic leads and the darker zone is the PI substrate. Fig. 1-b) shows the resistance, $R$ (thick line, left axis), and the vertical displacement, $\mathrm{z}$ (thin line, right axis), of the inner loading bars of the 4-point bending bridge (4PBB) as a function of time. The 4PBB is operated 
in a quasi-static mode (velocity of the loading bars $v=1 \mathrm{~mm} / \mathrm{min}$ ) during a four-cycles loading experiment. The microresistors stand in the region between the inner loading bars of the 4PBB, on the tensile surface of the specimen. In that region the longitudinal strain in the film as a function of the displacement of the bars, assuming that the neutral plane of the sensor is symmetrically placed between the two free surfaces of the substrate, is given by [10]:

$$
\varepsilon_{x x}=\frac{3 d_{s} z}{(3 l-4 a) a}
$$

where $l$ is the distance between the 4PBB outer loading bars and $a$ is the distance between the first and second loading bars. In our set-up $l=25 \mathrm{~mm}$ and $a=7.5 \mathrm{~mm}$. Using eq.(1), the resistance (Agilent 34401A digital multimeter) of the microresistor can be plotted as a function of the applied strain, which is done in fig.1-c). The slope of the graph is the longitudinal $G F$ of the $\mathrm{n}^{+}-\mathrm{nc}-\mathrm{Si}: \mathrm{H}$ sensing element. Here, longitudinal means that resistor current and applied strain are parallel. From the figure, $G F=-28.1$. Notice the minus sign of the $G F$, meaning that the resistance of the sensor decreases (increases) when tensile (compressive) strain is applied to the substrate. Hence the phase opposition that is patent between the two graphs of fig.1-b).

Fig. 2 shows the time-domain results of the dynamic actuation of large-area sensors. Each piezoresistor, $R_{\mathrm{S}}$, in the sensor is connected through the pads to a Wheatstone bridge external circuit in a quarter-bridge configuration and to a NI-USB 6210 data acquisition module connected to a PC laptop. The Wheatstone bridge is operated as close as possible to its maximum sensitivity point $\left[r=1\right.$ in eq.(2)], using two $27 \mathrm{k} \Omega$ resistances, a value close to $R_{\mathrm{S}}$. A variable resistor, $R_{4}$, is used to set the bridge into equilibrium $\left(V_{\text {out }}=0 \mathrm{~V}\right)$ while the sensor is 
in the flat, unstrained condition $\left(R_{\mathrm{S}}=R_{0}\right)$. The output voltage of the sensor under applied strain is the unbalanced $V_{\text {out }}$ of the bridge. No filtering, amplification, or other type of signal conditioning is used. $V_{\text {out }}$ is related to the relative resistance change of the piezoresistor by [11]:

$$
V_{\text {out }}=\frac{r}{(1+r)^{2}} \frac{\Delta R_{S}}{R_{0}} V_{I N}
$$

where $r=27 \mathrm{k} \Omega / R_{4}$ and $V_{I N}=5 \mathrm{~V}$ is the voltage supplied to the bridge by the USB bus.

Dynamic tests were performed using a Frederiksen Vibration Generator driven by a Function Generator that applied forced sinusoidal oscillations to its axle in the range 0.1-100 Hz. The rectangular sensor is clamped to the vibrating axle of the Generator by one of its smaller edges in a cantilever configuration. Data acquisition rate was set to $1000 \mathrm{~s}^{-1}$.

Fig.2-a) shows $V_{\text {out }}$ for the entire $86.775 \mathrm{~s}$ time interval that lasted the experience. During the first $37.7 \mathrm{~s}$ the oscillator axle was moving up and down at a frequency $f=0.3 \mathrm{~Hz}$. At this quasi-DC frequency the sensor basically executes translation following the axle where it is fixed and does not vibrate. In this region the sensor signal is essentially noise. The two bumps centered at $t=15 \mathrm{~s}$ and $t=25 \mathrm{~s}$, respectively, are the response to large deflections up (compression) and down (traction) imposed to the sensor with the operator finger, as a test. For $t>\sim 40 \mathrm{~s}$ the sensors start to oscillate at the frequency of the excitation but the amplitude of the signal $V_{\text {out }}$ strongly changes at some frequencies. If one concentrates on the transition that occurs in $V_{\text {out }}$ between $t \sim 49 \mathrm{~s}$ and $51 \mathrm{~s}$ [fig.2-b)] and compares to the constant-amplitude

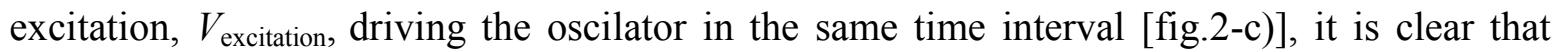
while the frequency of excitation decreases from $\sim 55 \mathrm{~Hz}$ to $34.3 \mathrm{~Hz}$, the amplitude $V_{\text {out }}$ 
increases (compressive side) from $\sim 2 \mathrm{mV}$ to $V_{\text {out }} \sim 30 \mathrm{mV}$. The reason is that the plastic substrate has a mechanical resonance mode at this frequency. This fact was visible to the naked eye and was confirmed by finite element simulation. Fig.2-d) shows the signals recorded at the resonance condition from three piezoresistors in the sensor (channels 1 to 3 ) situated at three different distances from the clamped edge of the sensor, along a longitudinal axis. The ratio of the signal amplitudes $(1: 0.54: 0.15)$ is the same as the ratio between the strains felt by the three piezoresistors at the resonance, according to the finite element simulation of the resonant mode. These results demonstrate the accuracy of the sensor as a strain-voltage transducer.

In moving from test structures with a few resistors to arrays of sensors the readout electronics should be re-designed. The most common techniques of measuring resistance changes are based either on four wires or in Wheatstone bridges (see above). However, these techniques are not suitable when the resistive elements are disposed into a matrix form. On the other hand, with a two wires technique, it is possible to perform the readout of a resistive matrix without switching elements (e.g. MOSFETs) inside it, which greatly simplifies the fabrication process. In this technique, a voltage is applied to each row and the current is measured in each column. The readout can then be performed by scanning the array using a mutiplexer on each row and column of the matrix. The digital data is then processed, interpreted and stored internally by an ultra low-power micro controller, also responsible for the communication of two-way wireless data, e.g. from inside to outside the human body in case of invasive applications. Fig. 3-a) shows the readout circuit for a $4 \times 4$ matrix, where the switches are connected to read $R_{11}$. The matrix columns that are not being read must be connected to 
ground (and not to high impedance). As the impedance of the current-to-voltage converter, formed by $A_{1}$ and $R_{\mathrm{f}}$ in fig.3-a), is very low, $R_{12}, R_{13}$ and $R_{14}$ are shunted and their currents are null. Otherwise, the currents of these resistors would affect the measurement of $R_{11}$. The currents of $R_{21}, R_{31}$ and $R_{41}$ are not null, but they flow directly to the ground. These currents cause a voltage drop in the switch and thus an error in the measurement of $R_{11}$. This error increases with the resistance of the switch, so, it must be kept as low as possible.

The piezoresistive sensor matrix output signals are multiplexed in time and then converted to digital by the analogic-digital converter (ADC) [see fig. 3-b)]. Afterward, they are read by the central processing unit (CPU), which sends them to the radio-frequency (RF) module, in order to be transmitted to a remote computer. The CPU, ADC and RF module are internal components of a microcontroller, as well as the input/output (I/O) interface, which is used to control the multiplexers.

\section{Conclusions}

Strain sensors based on the piezoresistive property of $\mathrm{n}^{+}-\mathrm{nc}-\mathrm{Si}: \mathrm{H}$ thin film microresistors were fabricated by HWCVD at $150{ }^{\circ} \mathrm{C}$ on plastic substrates using standard lithographic techniques. For large-area devices the piezoresistive response was converted in an output voltage by use of a Wheatstone bridge circuit in the quarter-bridge configuration. $V_{\text {out }}$ thoroughly monitored the mechanical oscillations imposed to the sensor and accurately detected its resonance frequency peak, confirmed by finite element analysis. Microresistors, with gauge factor -28.1 , followed the low-frequency oscillations of a four-point bending bridge. A two-wire readout circuit was designed to operate the sensor array without switching elements. Data will be subsequently 
processed and stored internally by a micro controller, also responsible for the communication of two-way wireless data.

\section{Acknowledgements}

This work was supported by Portuguese Foundation for Science Technology - FCT under projects NANO/NMed-SD/0156/2007 and PTDC/CTM/66558/2006. V.C. and E.S.M. acknowledge FCT for PhD grants SFRH/BD/48708/2008 and SFRH/BD/46740/2008, respectively. 


\section{References}

1. C. S. Smith, Phys. Rev. 94 (1954) 42.

2. W. G. Pfann and R. N. Thurston, J. Appl. Phys. 32 (1961) 2008.

3. S. Middelhoek and S. A. Audet, Silicon Sensors. Delft, The Netherlands: Delft Univ. Press, 1994, pp. 95-193..

4. J. Bartholomeyczik, S. Brugger, P. Ruther, O. Paul, IEEE Sens. J. 5 (5) (2005) 872.

5. S. Nishida, M. Konagai, K. Takahashi Jpn. J. Appl. Phys. 25 (1986) 17.

6. P. Alpuim, V. Chu, J.P. Conde, IEEE Sens. J. 2 (4) (2002) 336.

7. J. P. Conde, P. Brogueira, V. Chu, Philosophical Magazine B 76 (3) (1997) 299.

8. G. Willeke, in: Jerzy Kanicki (Ed.), Amorphous \& Microcrystalline Semiconductor Devices, Vol. II, Artech House, Inc., Norwood, Massachussets, 1992, pp. 55-88.

9. P. Alpuim, J. Gaspar, P. Gieschke, C. Ehling, J. Kistner, N.J. Gonçalves, M.I. Vasilevskiy, O. Paul, oral communication to EMRS Spring Meeting 2010, Symposium A, Congress Center Strasbourg, France, June 7-11, 2010, submitted to Sensors and Actuactors A.

10. S. P. Timoshenko, Strength of Materials. Malabar, FL: Krieger, 1958, p. 93, 96, 218.

11. P. Horowitz and W. Hill, 1989, The Art of Electronics, $2^{\text {nd }}$ Ed., Cambridge University Press, Cambridge, UK. 


\section{List of figures captions}

Fig. 1 Piezoresistive response, under 4-point bending loading cycles of one nc-Si:H microresistor with dimensions $W \times L=100 \times 100 \mu \mathrm{m}^{2}$ and thickness $120 \mathrm{~nm}$ on a $125 \mu \mathrm{m}$ thick polyimide substrate. a) Optical micrograph of sensor and metallic leads; b) Sensor resistance, $R$ (left axis) and vertical displacement of loading bars, $z$ (right axis) as a function of time; c) Sensor resistance, $R$, as a function of strain, $\varepsilon$, calculated from data in b) using eq.(2). The slope is the $G F(-28.1)$.

Fig. 2 Time-domain results of the dynamic actuation of large-area sensors. Each piezoresistor, $R_{\mathrm{S}}$, in the sensor is part of a Wheatstone bridge circuit in a quarter-bridge configuration. In a) the output voltage of the sensor (the unbalanced $V_{\text {out }}$ of the bridge) is displayed. In b) a detail of graph a), between 49 and $51 \mathrm{~s}$, is zoomed in. In c) the constant-amplitude, variable

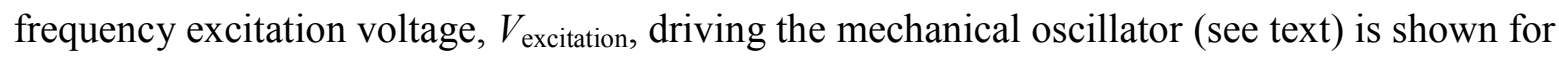
the time interval $[49,51] \mathrm{s}$. In d) the signal amplitudes and their ratio $(1: 0.54: 0.15)$ given by three piezoresistors in the sensor (channels 1 to 3 ) located at different distances from the clamped edge of the sensor.

Fig. 3-a) Readout circuit for a $4 \times 4$ sensor array. The switches are connected in the right position for reading $R_{11}$. In this case, a voltage is applied to $R_{11}$ and its current is measured. $R_{12}, R_{13}$ and $R_{14}$ are shunted, so their currents are null. b) Block diagram of the sensor electronic interface. 
Figure 1
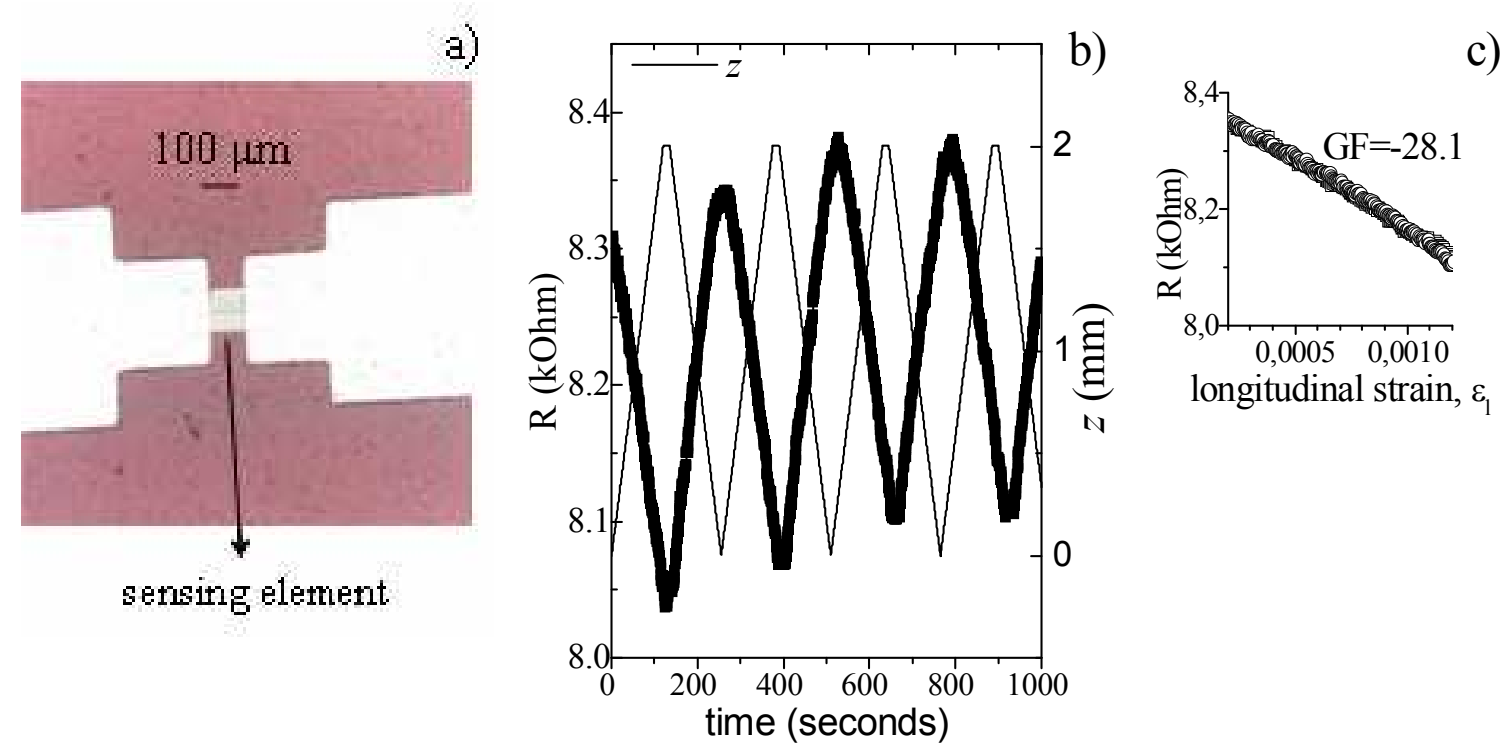
Figure 2

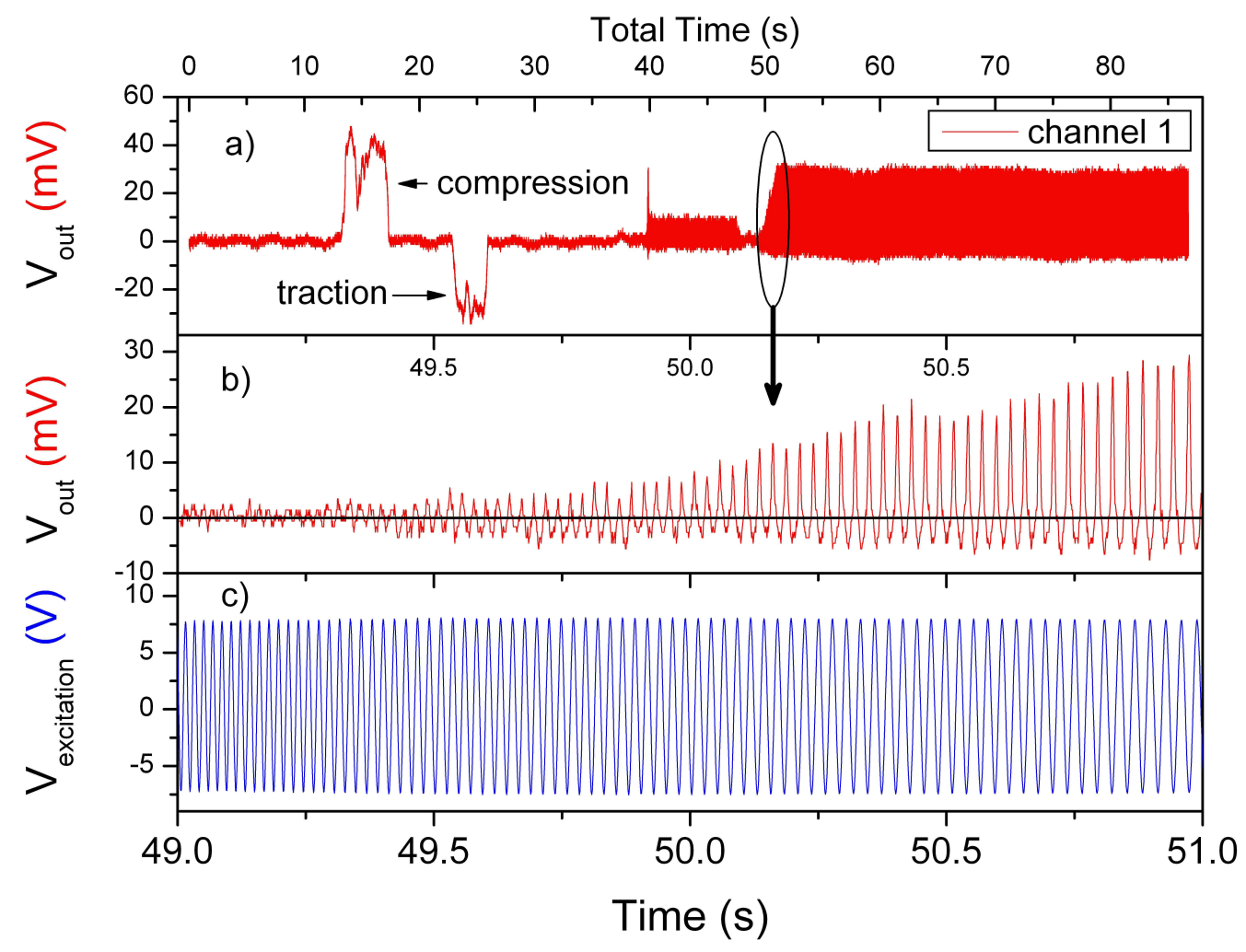

d)

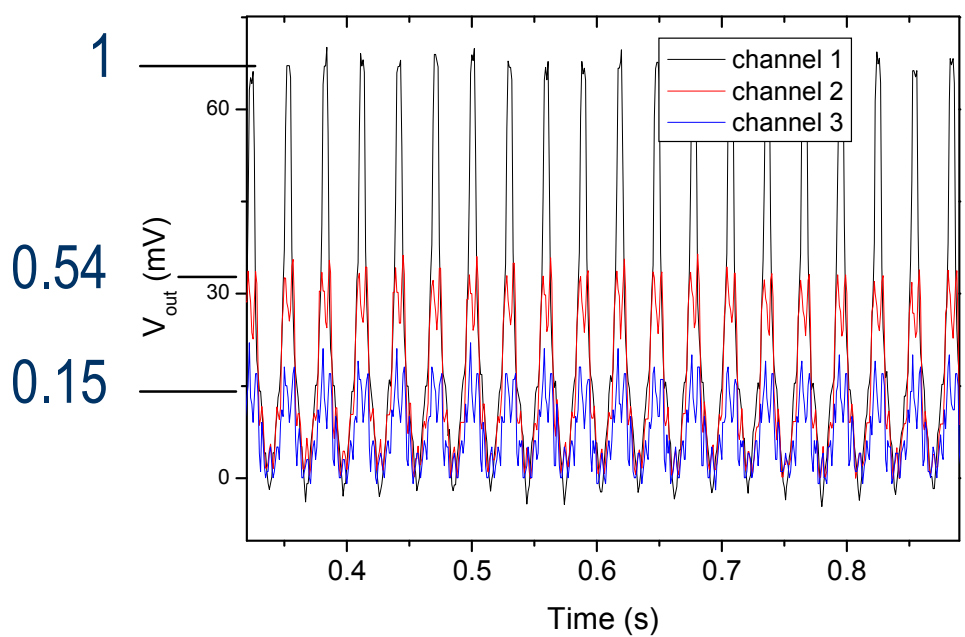


Figure 3
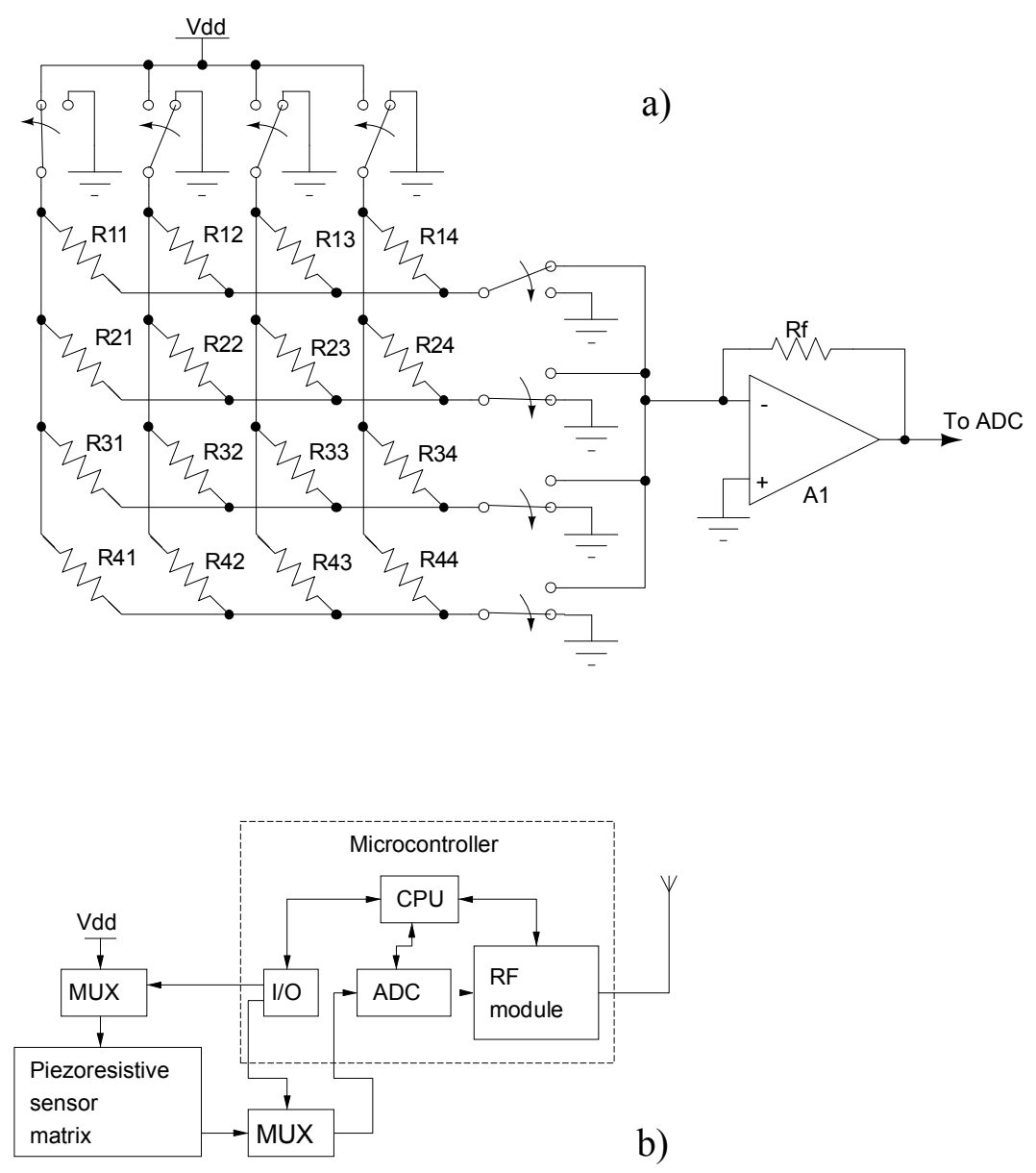